\title{
EVALUACIÓN DE LA TRANSFORMACIÓN DE AGUAS RESIDUALES EN PRODUCTOS DE VALOR AGREGADO: PRODUCCIÓN DE POLIHYDROXYALKANOATES (PHAS)
}

\author{
Evaluation of the transformation of wastewater into value-added products: Production of \\ polyhydroxyalkanoates (PHAs)
}

\author{
Jeniffer Gracia \\ jeniffer.Gracia@campusucc.edu.co \\ Jhessica Mosquera \\ Jhessica.mosquera@usantotomas.edu.co \\ Carlos Montenegro \\ cemontenegrom@udistrital.edu.co \\ Luisa Suarez \\ Lfsuarez3@poligran.edu.co \\ Paola Acevedo \\ paola.acevedop@ucc.edu.co \\ Iván Cabeza \\ icabeza@poligran.edu.co \\ Politécnico Grancolombiano \\ Ingeniería Industrial \\ Colombia
}

\begin{abstract}
Resumen
Los procesos de bioconversión se presentan como una oportunidad para alcanzar la sostenibilidad ambiental, teniendo como pilar principal la producción de combustibles, sustancias químicas y materiales de base biológica; los cuales pueden resultar del aprovechamiento de residuos. En esta búsqueda, se encontró que los lodos de plantas de tratamiento de aguas residuales (PTAR) constituyen un residuo altamente contaminante para el cual se requiere implementar alternativas de valorización. Entre las tecnologías aplicables, se encuentra la producción de polímeros biodegradables del tipo polihidroxialcanoatos (PHA), los cuales se pueden obtener a partir de cultivos microbianos mixtos que aprovechan líquidos fermentados ricos en ácidos grasos volátiles (AGVs). Por consiguiente, uno de los objetivos de este proyecto es encontrar las mejores condiciones para la producción de AGVs, que permitan el uso de lodos provenientes de la PTAR EL SALITRE en procesos futuros de generación de PHAs. Durante el desarrollo de la fase inicial del proyecto, se evaluaron diferentes condiciones operativas que afectan la producción de AGVs, como: carga orgánica, con niveles de 4 a $6 \mathrm{~g} \mathrm{SV} \mathrm{L}^{-1}$ y pH alcalino, entre 9.5 y 11.5. Los resultados obtenidos permitieron evidencias que lodos evaluados permiten obtener altas cargas de AGVs entre 1063 y 1300 AGV L $^{-1}$; en consecuencia, el líquido fermentado obtenido puede ser utilizado como fuente de carbono para la producción de PHAs.
\end{abstract}

Palabras clave:

Ácidos grasos volátiles, valorización de residuos, bio-procesos, polímeros biodegradables 


\section{Introducción}

La problemática relacionada con el sector plástico a nivel mundial ha hecho que el desarrollo de alternativas basadas en fuentes renovables y que presenten propiedades fisicoquímicas similares a los materiales generados por la industria petroquímica sea una prioridad para la comunidad científica nacional e internacional. En el país, existen diversos materiales considerados biopolímeros, que presentan características particulares y con usos específicos. Sin embargo, los polihidroxialcanoatos (PHA) son considerados únicos debido a su método de obtención, dado que se pueden producir a partir materias primas renovables y/o residuales y a la posibilidad de integración con rutas energéticas de valorización de biomasa como la co-digestión anaerobia bajo el concepto de biorefinerías (Kumar, y otros, 2019). Estos materiales, tienen características similares a la de los plásticos tradicionales cómo su baja solubilidad en agua, ductilidad y resistencia. Estos procesos, normalmente, tienen costos de producción superiores a los de plásticos tradicionales, lo que hace poco competitiva su comercialización (Mengmeng, Hong, Qingliang, Ngai Shirley, \& Jie, 2009). No obstante, en los últimos años, se han intentado desarrollar alternativas de producción de PHAs que incluyen la utilización de cultivos microbianos mixtos empleando sustratos residuales como fuente de carbono, con el fin de reducir los costos operativos y de inversión para la producción y comercialización en escala industrial de este tipo de material polimérico (Valentino, y otros, 2016). En este sentido, el presente proyecto hará uso del concepto de biorefinería con el fin de generar rutas de valorización de lodos de aguas residuales para la producción de ácidos grasos volátiles (AGVs), los cuáles serán empleados como fuente de carbono para la producción sostenible de PHAs.

\section{Método}

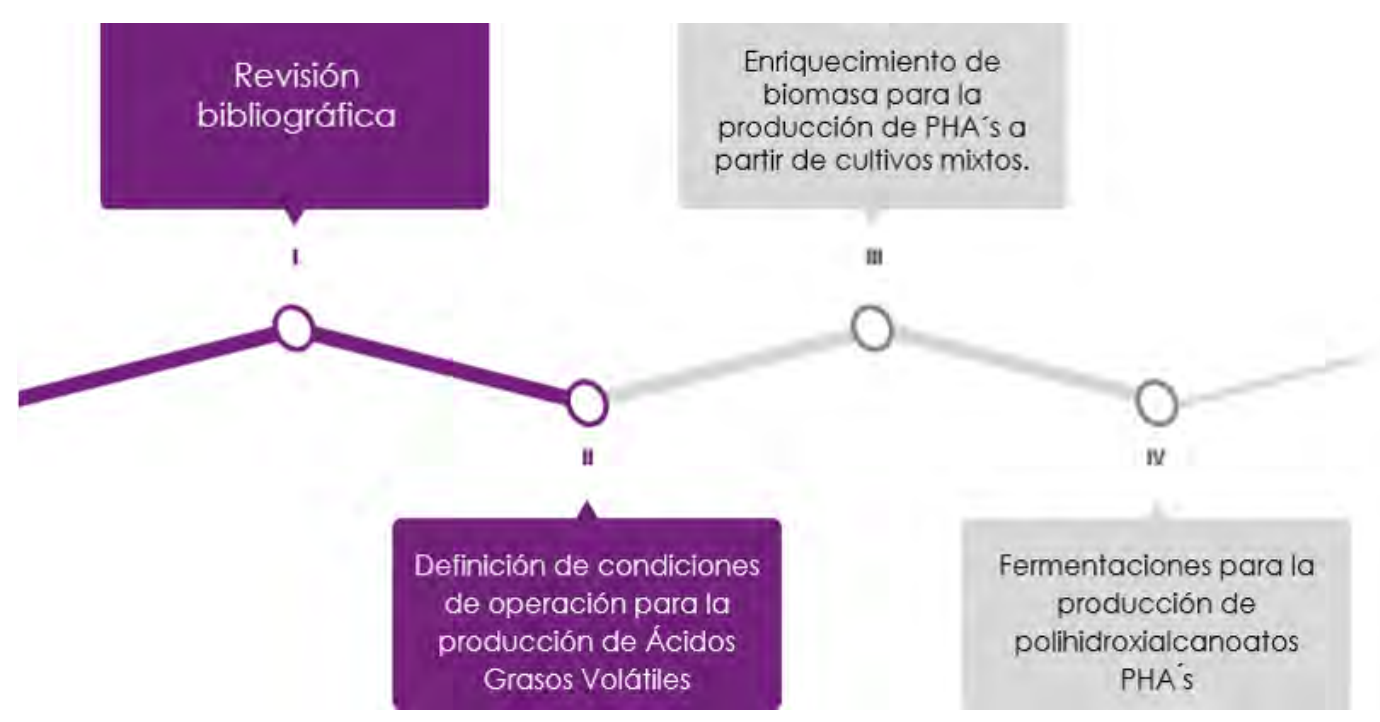


Con el objeto de definir las condiciones de operación para la producción de AGVs, se planteó un diseño experimental que permitiera la evaluar diferentes variables operacionales que afectan la producción de los ácidos grasos volátiles. Estas variables son: carga orgánica en gramos de solidos volátiles (g SV), con niveles entre 4 y 6 g SV L ${ }^{-1} ; \mathrm{pH}$ alcalino, entre 9.5 y 11.5. La carga orgánica fue ajustada a partir de de las características fisicoquímicas de la materia prima, lodos de aguas residuales de la PTAR El Salitre y el pH fue ajustado con una solución de $\mathrm{NaOH}$ al 0.5 M(C. Daza-Orozco, 2015; C E Daza-Orozco, 2015; Daza-Orozco E.; Ramírez-Varela, F.; Arboleda-Muñoz, G.; Gil-Ángel, G.; Villada-Castillo, H.; Portela-Guarín, H.; García-Fernández, J.; Estévez-Ceballos, J.; Infante- Castillo, A.; Rodríguez-Cuberos, E.; Cascante-Gatgens, A.; Barrien, M., 2019).

El proceso de fermentación se llevó a cabo en reactores tipo batch, con un volumen de trabajo de $200 \mathrm{~mL}$, los cuales se ubicaron en un baño termostatizado para garantizar condiciones mesofílicas $\left(35 \pm 1{ }^{\circ} \mathrm{C}\right)$ durante 12 días consecutivos (Figura 1). La producción de AGVs fue evaluada a través del seguimiento cada tres días, durante 12 días de fermentación acidogénica, las muestra obtenidas se sometieron a una caracterización de acuerdo a métodos estándar.

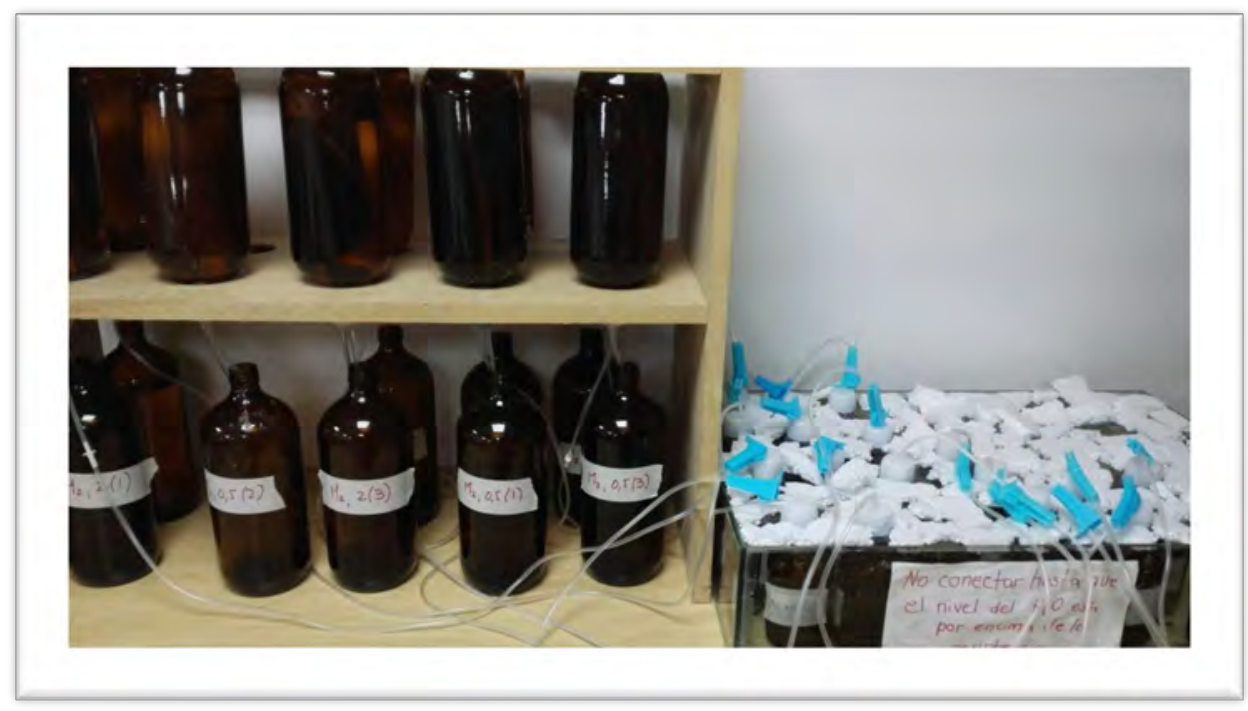

Figura 1. Fermentación acidogénica en escala de laboratorio.

Los líquidos fermentados obtenidos, ricos en AGVs, permitieron desarrollar una primera evaluación del proceso de acumulación de PHA. El proceso de acumulación de PHA se realizó en Erlenmeyer de 500 mL, que se llenaron a un $30 \%$ de su capacidad y manejaron condiciones aerobias a una temperatura de $25^{\circ} \mathrm{C}$. El líquido fermentado fue alimentado periódicamente después de un proceso de estabilización microbiológica. Se realizó seguimiento de la acumulación del biopolímero cada 12 horas.

\section{Resultados}

La caracterización de lodos de la PTAR El Salitre cuenta con las siguientes características fisicoquímicas: $11.39 \pm 0.06 \mathrm{~g} \mathrm{SV} \mathrm{L}^{-1}, 125.5 \mathrm{~g} \mathrm{DQO} \mathrm{L}^{-1}$ y $0.11 \pm 0.01 \%$ de nitrógeno total. Esta caracterización permitió ajustar las variables propuestas en el diseño experimental, los resultados del proceso experimental se resumen en la Figura 2. 


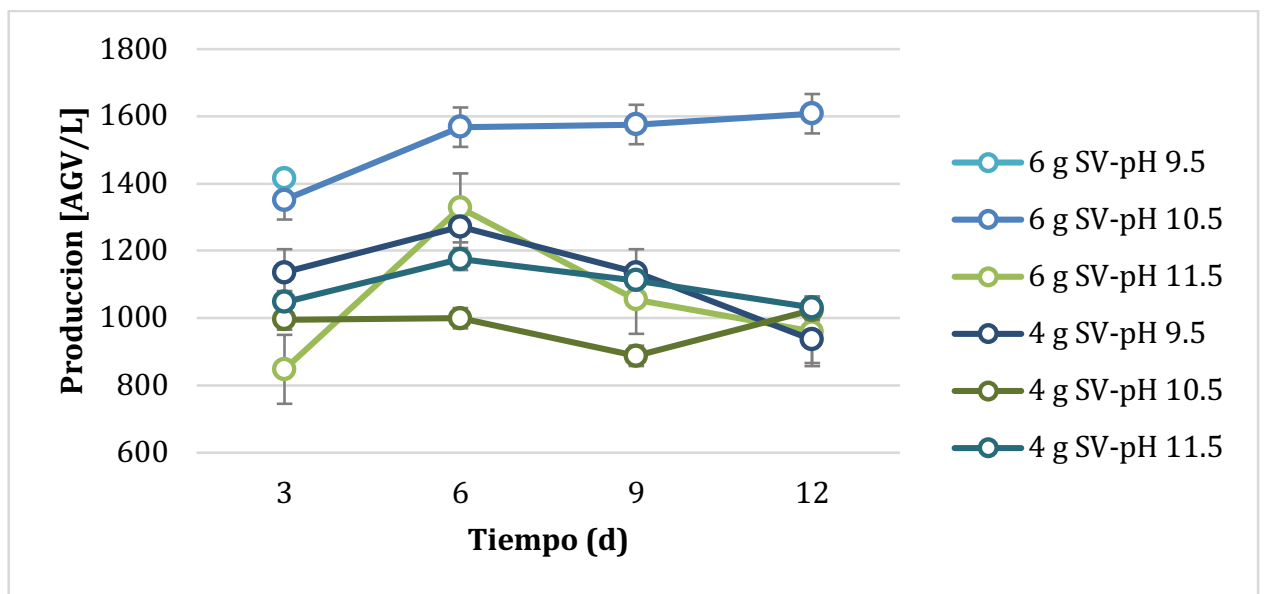

Figura 2. Comportamiento en la producción de ácidos grasos en 12 días de fermentación del lodo.

\section{Discusión y Conclusión}

Los perfiles de producción para las condiciones de tratamiento propuestas demuestran que en todas las condiciones de tratamiento el proceso fermentativo se inicia de inmediato, registrando incrementos en la producción de AGV desde el primer día de seguimiento. En los procesos de fermentación acidogénica, altas cargas orgánicas pueden ser usadas para la producción de AGVs y disminuir el tiempo de fermentación (Yuan, y otros, 2019), los resultados obtenidos muestran que las producciones altas de AGV se presentan bajo cargas orgánicas de $6 \mathrm{~g} \mathrm{SV} \mathrm{L}^{-1} \mathrm{y} \mathrm{pH}$ de 10.5 manteniendo estable el proceso y evitando la acidificación del sistema.

En la Figura 3. se observa el proceso de fermentación aerobia bajo condiciones controladas de temperatura, al final de la fermentación, se pudo apreciar un cambio en la densidad del caldo de cultivo, lo cual se alude al proceso de acumulación de PHA. Actualmente se desarrollan nuevos ensayos que permitirán la cuantificación másica de acumulación de biomasa y biopolímero.

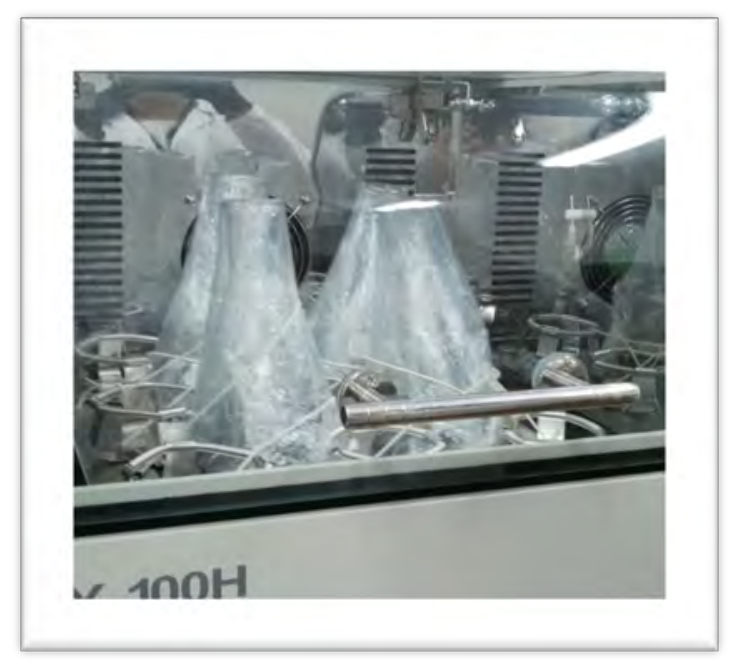

Figura 3. Proceso de acumulación de PHA, fermentación aerobia bajo condiciones controladas de temperatura. 
El desarrollo completo del proyecto tendrá los siguientes impactos sobre la industria, la sociedad y el ambiente.

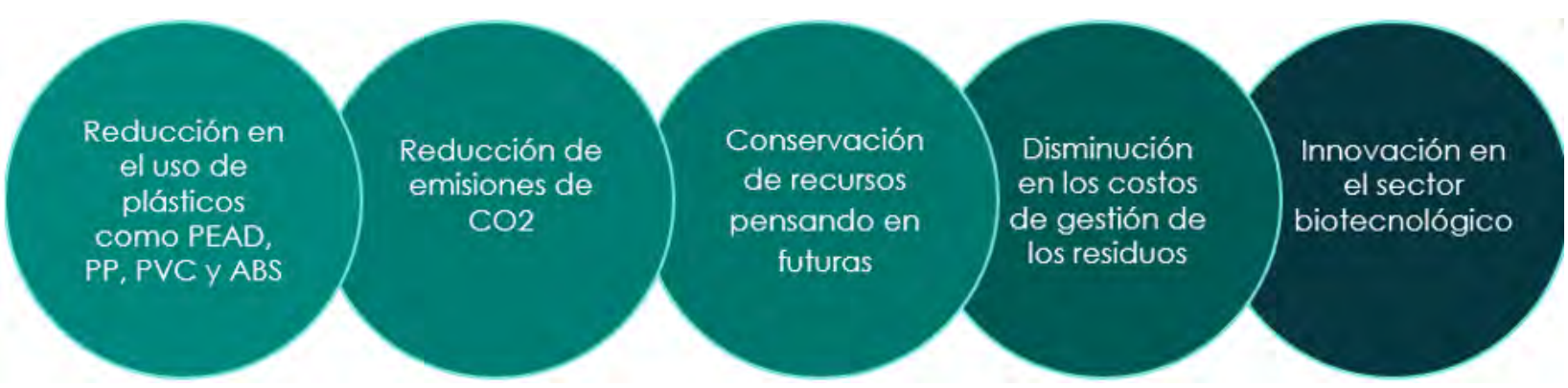

Referencias bibliográficas

Daza-Orozco, C. (2015). La Investigación como proyecto de Vida: Un acercamiento al quehacer de los Semilleros de Investigación. https://doi.org/10.13140/RG.2.1.2631.1761

Daza-Orozco, C E. (2015). La Investigación en los tiempos del acceso abierto. Silogismo. Retrieved from http://www.cide.edu.co/ojs/index.php/silogismo/article/view/294

Daza-Orozco E.; Ramírez-Varela, F.; Arboleda-Muñoz, G.; Gil-Ángel, G.; Villada-Castillo, H.; Portela-Guarín, H.; García-Fernández, J.; Estévez-Ceballos, J.; Infante- Castillo, A.; Rodríguez-Cuberos, E.; Cascante-Gatgens, A.; Barrien, M., C. E. . N.-A. (2019). Iniciación científica: conceptualización, metodologías y buenas prácticas (1st ed.; Carlos Eduardo Daza-Orozco, Ed.). https://doi.org/10.13140/RG.2.2.20812.23684

Kumar, G., Ponnusamy, V., Bhosale, R., Shobana, S., Yoon, J., Bhatia, S., . . . Kim, S. (2019). A review on the conversion of volatile fatty acids to polyhydroxyalkanoates using dark fermentative effluents from hydrogen production. Bioresource Technology.

Mengmeng, C., Hong, C., Qingliang, Z., Ngai Shirley, S., \& Jie, R. (2009). Optimal production of polyhydroxyalkanoates (PHA) in activated sludge fed by volatile fatty acids (VFAs) generated from alkaline excess sludge fermentation. Bioresource Technology.

Valentino, F., Morgan-Sagastume, F., Campanari, S., Villano, M., Werker, A., \& Majone, M. (2016). Carbon recovery from wastewater through bioconversion into biodegradable polymers. New Biotechnology.

Yuan, Y., Hu, X., Chen, H., Zhou, Y., Zhou, Y., \& Wang, D. (2019). dvances in enhanced volatile fatty acid production from anaerobic fermentation of waste activated sludge. Science of the Total Environment. 